\title{
A remote and immersive setup for pandemic-safe surgical education
}

Jean-Claude Rosenthal, Fraunhofer Heinrich-Hertz-Institut HHI, Berlin, Germany jean-claude.rosenthal@hhi.fraunhofer.de, Armin Schneider, Munich Surgical Imaging GmbH, Munich, Germany, aschneider@munichimaging.de, Eric L. Wisotzky, Fraunhofer Heinrich-Hertz-Institut HHI, Berlin, Germany eric.wisotzky@hhi.fraunhofer.de, Senna Meij, TU Delft, Department of BioMechanical Engineering, Delft, Netherlands, s.e.p.meij@tudelft.nl, John van den Dobbelsten, TU Delft, Department of BioMechanical Engineering, Delft, Netherlands, J.J.vandenDobbelsteen@tudelft.nl, Philipp Arens, Charité Universitätsmedizin, Berlin, Germany, philipp.arens@charite.de, Florian C. Uecker, Charité Universitätsmedizin, Berlin, Germany, fc.uecker@charite.de

\section{Introduction}

Existing challenges in surgical education (See one, do one, teach one) as well as the Covid-19-pandemic make it necessary to develop new ways for surgical training. This is also crucial for the dissemination of new technological developments. As todays live transmissions of surgeries to remote locations always come with high information loss, e.g. depth perception, this work describes the implementation of a scalable remote solution for surgical training, called TeleSTAR, using immersive, interactive and augmented reality elements tested during 3 ENT surgeries.

\section{Methods}

The system uses a full digital surgical microscope (ARRISCOPE, Munich Surgical Imaging, Munich, Germany). The microscope has a modular software augmented reality interface, which consists of an interactive annotation mode to mark anatomical landmarks using an integrated touch panel as well as an intraoperative image-based stereo-spectral algorithm unit to measure and highlight anatomical details and tissue characteristics. The stereo measurement unit computes a complete depth map allowing to perform precise distance measurements by setting markers. In addition, the system comes with a bi-lateral audio pipeline to foster direct communication.

\section{Results}

We evaluated the new educational paradigm during the 3D broadcast of three live AR-based cochlea implant surgeries. The system scaled to five different remote locations with low latency and offering a delayed 2D YouTube stream. In total, more than 150 persons could be trained including healthcare professionals, biomedical engineers and medical students. It was demonstrated by a participants survey, that annotated 3D live transmissions improve the learning outcome by adding an extra level of surgical transparency.

\section{Conclusion}

TeleSTAR provides a highly scalable solution for surgical training solving limitations for current educational programs and during pandemic situations by using an adaptive combination of modular software and hardware modules. Furthermore, it can be used as an intraoperative assistance system by support from remote experts and it can be easily adapted to other surgical domains. 


\section{Extreme Constructive Alignment: How Multimodal Examinations can Increase Sustainable Student Gain by Aligning Teaching and Assess- ment}

Ulrich M. Engelmann, Department of Medical Engineering and Applied Mathematics, FH Aachen University of Applied Sciences, Aachen/Jülich, Germany / enmodes GmbH, Aachen, Germany, engelmann@fh-aachen.de Michael Gundlach, Department of Ecosystem Analysis, Institute for Environmental Research, RWTH Aachen University, 52074 Aachen, Germany, m.gundlach@fz-juelich.de Martin Baumann, Institute for Applied Medical Engineering, Helmholtz-Institute, RWTH Aachen University Hospital and RWTH Aachen University, Aachen, Germany, baumann@ame.rwth-aachen.de

\section{Introduction}

What does the future biomedical engineering graduate need for a successful transition into industrial career? Modern industry and multi-discipline projects require highly trained individuals with resilient science and engineering backgrounds. These graduates must be able to agilely apply excellent theoretical knowledge in their subject matter as well as essential practical "hands-on" knowledge of diverse working processes to solve complex problems. To meet these demands placed on graduates, university education follows the concept of Constructive Alignment and thus increasingly adopts the teaching of necessary practical skills to the actual industry requirements and respective assessment routines. However, a systematic approach to coherently align these three central teaching demands is strangely absent from current university curricula.

\section{Methods}

We demonstrate the feasibility of implementing practical assessments in a regular theory-based examination, thus defining the term "blended assessment". We assessed a course for natural science and engineering students, mostly pursuing a career in the biomedical field, and evaluated the benefit of blended assessment exams for students and lecturers. Our controlled study assessed the physiological background of electrocardiograms (ECGs), the practical measurement of respective ECG curves, and their interpretation for normal and basic pathologic alterations. To study on long time effects, students have been assessed on the topic twice with a time lag of 6 months.

\section{Results}

Our findings suggest a significant improvement in student gain with respect to practical skills and theoretical knowledge thereof. The results of the reassessments support these outcomes.

\section{Conclusion}

From the lecturer's point of view, blended assessment complements practical training courses while keeping organizational effort manageable. We consider blended assessment a viable tool for providing an improved student gain, industryready education format that should be evaluated and established further to prepare university graduates optimally for their future careers. 


\section{How to Support Students in Maximizing their Outcome with Online Cooperation?}

Mark J. Benninghoff, Institute for Applied Medical Engineering, Helmholtz-Institute, RWTH Aachen University Hospital and RWTH Aachen University, Aachen, Germany, benninghoff@ame.rwth-aachen.de

Peter Isfort, Clinic for Diagnostic and Interventional Radiology, RWTH Aachen University Hospital and RWTH Aachen University, Aachen, Germany, isfort@ukaachen.de

Martin Baumann, Institute for Applied Medical Engineering, Helmholtz-Institute, RWTH Aachen University Hospital and RWTH Aachen University, Aachen, Germany, baumann@ame.rwth-aachen.de

\section{Introduction}

During the last year, student cooperation has shifted to the digital space. Chats, videoconferencing and online collaboration tools replace physical meetings and face-to-face interactions. While many online tools provide sophisticated technologies to share screens and co-edit manuscripts, the support of collaborative thinking, learning and even fooling around is still underdeveloped, but remains a valuable resource.

This study traces whether the given level of instruction details influences the collaboration effectiveness among student working groups in a practical course in Biomedical Engineering.

\section{Methods}

Within the semester's practical course of Introduction into Medicine, participating students were divided into three groups (A, B and C) of each 4 to 6 students and had to collaborate on a clinical case. Each group was asked to to fulfil an additional pre- and post-task before and after the semester's practical course. These tasks consisted of solving combinatory puzzles based on isolated information bits and hints that were distributed among the group members. The groups differed only in the instructions they received to fulfil the task:

- Group A (11 in total) received two written pages with detailed hints and rules how to effectively collaborate online. These contained recommendations e.g. for creating an agenda, elect a member as moderator and to document every decision in a protocol.

- Group B (11) was instructed to create own rules before commencing with the main task, write them down and abide to them.

- Control group C (10) was only instructed with basic technical information to handle the session.

During their work phases, all groups were observed by a hidden and non-participating person, who noted collaboration data e.g number of interactions, collaboration stringency and time to success. The study was conducted twice (in the winter term with slightly younger students and in the summer term with slightly older students) with different student cohorts. Written informed consent was obtained from all students prior to the beginning of the respective practical course.

\section{Results}

After compiling the data, school grades were assigned representing 1 for the best up to 6 for the worst performance. In average we noticed that the groups B received the best grades (average 1,5 / median 1 for winter term and average 1,6 / median 1,5 for summer term). Second best performance was shown by the groups A (average 2,14 / median 2 for winter term and average 1,75/ median 2 for summer term) and finally groups $C$ performed worst (average 2.10 / median 2 for winter term and average 1,85/median 2 for summer term ). In the taken times to solve the puzzle tasks, groups $\mathrm{B}$ showed up to be best in the winter term and groups $\mathrm{A}$ in the summer term. In all categories groups $\mathrm{C}$ delivered the worst grades in average.

\section{Conclusion}

The results of this study support that it is most reasonable to aid student online collaboration by providing them with a few hints and instruct them to create their own rules for online collaboration (as done with group B). The results further support that the cooperation gets worse without suggesting any rules or instructions and that student age possesses a negligible influence. 


\section{State-of-the-Art: Biodesign based Innovation Ecosystems in Europe}

Holger Fritzsche, INKA HealthTec Innovation Laboratory, Medical Faculty, Otto-von-Guericke University, Magdeburg, Germany, holger.fritzsche@ovgu.de

Elaha Mahbub, Ethical Innovation Hub, Institute for Electrical Engineering in Medicine, Universität zu Lübeck, Lübeck, Germany

Axel Boese, INKA HealthTec Innovation Laboratory, Medical Faculty, Otto-von-Guericke University, Magdeburg, Germany, axel.boese@med.ovgu.de

Michael Friebe, INKA HealthTec Innovation Laboratory, Medical Faculty, Otto-von-Guericke University, Magdeburg, Germany, michael.friebe@,ovgu.de

\section{Introduction}

Today's healthcare challenges with unmet clinical needs, high regulation and certification standards, and increasing costs demand faster innovation and technical translation. To address this challenge, Stanford released a fellowship called Biodesign, where need-based healthcare innovation is taught with the approach identify, invent and implement. Since then, different European institutions have adopted the Biodesign innovation approach and organized within the Biomedical Engineering- Innovation, Design, and Entrepreneurship Alliance (BMEidea EU). The generation of successful healthcare innovation isn't only based on participating in an Innovation teaching program. It is much more a matter of having the right innovation ecosystem with experts, the respective stakeholders, and access to essential resources within reach.

\section{Methods}

Through a qualitative survey, seven BioDesign based teaching programs in the EU were examined. The study from an academic perspective contains information collected about Resources, Activities, Academic Performance, and Transfer Performance.

\section{Results}

The results from the survey are analysis regarding the healthcare innovation ecosystem. The results are described individually for each institution and each ecosystem category: Resources (employees, network partners, cooperation to industry and other research institutions), Activities (executed teaching programs, participants, identified unmet clinical needs), academic performance (research projects, papers and publications, and studies) and transfer performance (patents, number of ideas, prototypes, and identified unmet clinical needs passed on to the industry, founded Startups).

\section{Conclusion}

The demand for new healthcare innovations, and especially innovation training programs that address challenges, developed collaboratively with the respective stakeholders, is increasing. Additionally, there is a growing expectation that innovation needs to reach the market quickly and be implemented accordingly.

A Healthcare Innovation Ecosystem, where different entities function as a productive unit with a shared vision and committed to application-driven research and technology transfer, will increase innovation's success and adaptation. 


\section{Forecasting the Future of Healthcare Democratization}

Beatrice Barbazzeni, Institute of Cognitive Neurology and Dementia Research, Faculty of Medicine, Otto-von-GuerickeUniversity, Magdeburg, Germany, beatrice.barbazzeni@med.ovgu.de

Holger Fritzsche, INKA HealthTec Innovation Laboratory, Faculty of Medicine, Otto-von-Guericke-University, Magdeburg, Germany, holger.fritzsche@,ovgu.de

Michael Friebe, INKA HealthTec Innovation Laboratory, Faculty of Medicine, Otto-von-Guericke-University, Magdeburg, Germany, michael.friebe@ovgu.de

\section{Introduction}

Health longevity, cost reduction, prevention based healthcare, personalized medicine, predictive diagnostic, transformed care delivery, de-aging, healthy lifestyle trends, and implementation of smart technologies should lead to a more democratized healthcare provision. Forecasting the future of healthcare and health policy certainly is imperfect but a highly valuable scientific approach to innovation. In the last decade the healthcare system faced several challenges including significant increase of care costs, demographic changes towards the older population, inability to prevent and overcome pandemics, increased chronic and non-communicable diseases, or resistance to adopt emerging technologies. The need to shift the focus from sickness to health becomes a necessary mission.

\section{Methods}

We developed a lecture titled „Healthtec Innovation Design“ for graduate students from medicine, bioengineering, data science and software engineering. Lecture goal is to teach innovation methodologies, exponential technologies and methods to achieve healthcare democratization. An important component is to promote initiatives with global teams focused on the future of health.

\section{Results}

The educational and initiative programs were impactful in growing interest toward innovation focusing on disruption and healthcare democratization. Participants raised awareness and will to implement the taught skills and methods in their current project with enthusiasm and optimistic thinking. Individual statements from different viewpoints were qualitatively processed to generate a big picture.

\section{Conclusion}

With the advent of the fourth industrial revolution, the process of digitization has a great impact in our society leading to exponential innovations. Embracing digitalization, a patient-centric approach, affordable care services, and the expansion of precision medicine, the entire healthcare organization and management would undergo a worldwide change overcoming those challenges. Particularly, digital technologies, the leverage of artificial intelligence and empathy would satisfy unmet clinical needs. With a future oriented statement, the forecast of healthcare becomes now more imaginable, in which democratization will allow the affordability of services in different countries and economic status. 


\section{Is there a loss in quality between a whole-day face-to-face instructor-led seminar with group work and an instructor-led distance-learning alterna- tive?}

Maria Henke, Institute for Robotics and Cognitive Systems, Universität zu Lübeck, Lübeck, Germany, maria.henke@uniluebeck.de

\section{Introduction}

Driving digitisation, reaching an audience spread over different places and reduction of travel costs are reasons to change the delivery mode of face-to-face seminars and trainings to distance-learning alternatives. Since most people are used to face-to-face instruction and the conditions of face-to-face instruction differ essentially from the conditions of distancelearning instruction there was a strong reluctance to this change. A common opinion is that it involves a loss in quality, especially, when social learning such as group work is involved in the instructional concept. This work shows that the change from face-to-face to distance-learning instruction is possible without a loss of quality.

\section{Methods}

The evaluation of 19 seminars with the same content and learning objectives are compared, 13 of them were given as whole-day (eight hours incl. breaks) face-to-face instructor-led seminars with group work between 2013 and 2019 and six of them were delivered in two parts of four hours incl. breaks on two different days as instructor-led distance-learning seminar also including the same group work tasks between 2020 and 2021. The instructional concept was transferred carefully from the face-to-face setting to the distance-learning conditions. All seminars were given by two instructors; one instructor was the same in all but one seminars, the other changed through the years.

\section{Results}

The comparison shows that there is no loss in quality for the examined seminar.

\section{Conclusion}

No loss in quality could be shown, suggesting that the widespread fear that in any case a change from face-to-face to a distance-learning instructional setting is associated with a loss in quality is unfounded. 


\section{Software-based practical course on visualization and processing of medical images}

Ralf Hinderer, Faculty of Physical Engineering/Computer Sciences, Westsächsische Hochschule Zwickau, Zwickau, Germany, ralf.hinderer@fh-zwickau.de

\section{Introduction}

Nowadays images like CT or MRI images play an important role in many fields of medicine. They are usually of digital nature and can be visualized and processed with an appropriate software. For different reasons this aspect is rarely considered in the practical training of students of biomedical engineering and related subjects. To address this problem, a practical course on visualization and processing of medical images was developed at the Westsächsische Hochschule Zwickau (WHZ).

\section{Methods}

The practical course is based on the free, open source software package 3D Slicer. Most of the image data sets used were taken from 3D Slicer's website. Typically, a unit of the course takes three hours. The students, usually in their third year of undergraduate study, work through the exercises on their own or in small groups. The course takes place either in a computer room or on the internet using a web conferencing system.

\section{Results}

Currently, the course comprises five units covering topics such as visualization of medical images, image segmentation including 3D printing, image registration and DICOM. It is important to note, that the course instructor must know the software package very well und has to keep the course material up-to-date. Evaluations show that the course is appreciated and very well received.

\section{Conclusion}

Most of the students work with digital medical images for the first time and get practical experience with essential applications of image processing in medicine. In its fourth year, the course is well established at the WHZ and is an integral part of a wider approach to incorporate aspects of digitization into the curriculum of biomedical engineering. The possibility to teach the course online is an attractive option in pandemic times and for remote teaching in general. 